\title{
The Way We Deviate from Baseline
}

\author{
Yumiko Tozawa* \\ Department of Nephrology and Endocrinology, Applied Life Sciences, Japan
}

Received: 眥: November 14, 2018; Published: 鴊: November 26, 2018

*Corresponding author: Yumiko Tozawa, Department of Nephrology and Endocrinology, Tokyo, Japan

\begin{abstract}
Inflammation can begin acutely or slowly. Inflammation accompanies a number of biological processes in pathology and physiology. The reason why we often conclude based on the rate of initial development of an inflammatory disease that we are soon to be restored to our basal conditions, safe and sound, remains unknown. Here, I present a hypothetical model of an 'information'-generating system for the body and cell based upon an assumption that our immediate response to external stimuli consists of bodily and cellular responses. Upon the initiation of every stimulus, body and cell must deviate from baseline and then return to baseline. How much the body and cell can deviate from baseline is determined by the capacity of an 'information'-generating system. The rate of increase in nitric oxide availability in a compartment may be a discriminating factor for the capacity of a cellular 'information'-generating system.
\end{abstract}

\section{Introduction}

\section{Part One}

Inflammation is manifested during the course of almost all disease states. However, in some cases, inflammation can be recognized as a physiological process. It seems quite difficult to clearly identify inflammatory processes as physiological or pathological. This identification is exemplified in angiogenesis, which shares many aspects with inflammation. Angiogenesis occurs in the yolk sac, preceding all other processes of organogenesis; it accompanies recovery from traumatic injuries, and it is an aspect of the pathology of diseases as a process toward or a component of complications. We cannot live a complete life if we avoid inflammatory processes. In general, there is a case for acutely beginning inflammatory diseases of a good and fast recovery when there is a benign nature of diseases; however, we do not have such an outlook in the case of slowly progressing diseases. Strangely enough, a reason why we do and do not remains unclear. It has to be of interest why the hope for a fine and fast recovery can be abandoned if an inflammatory disease begins slowly and why we can conclude, based on the initial rate of disease development, that the nature of the disease will remain benign. We must have automatically recognized a difference between acutely beginning and slowly beginning diseases that should be reflected in a difference in numerical values for variables functionally dependent on time at a given time point near the very beginning of an inflammatory disease. Our constant automatic reaction to every prognosis must have been long cherished and supported by the truth of Nature and must be a clue to unraveling a true mechanism that leads inflammatory processes to various destinations.

\section{Part Two}

Life is inspired by external stimuli. Living begins with the acquisition of responsiveness to stimuli; life develops, declines and eventually terminates with the loss of responsiveness to stimuli. We cannot live even for a moment if we avoid immediate (short-term) responses to stimuli. Immediate responses to stimuli are the most basic feature that is important to survival through every moment of life since we cannot defend our lives against foreign threats without immediate responses. Multicellular organisms function as if they were unicellular organisms. A multicellular organism builds, as a whole, an immediate response to an external stimulus. Each cell of a multicellular organisms, be that as it may, would potentially keep its independence to build an immediate response to a stimulus. The degree of independence differs from cell to cell. A greater degree is generally seen in the cells of mesodermal origin and is most typically exemplified in blood cells. These cells include red blood cells and platelets without nuclei. Immediate responses to stimuli must start and terminate independently of genomes.

Our immediate responses to stimuli must include two stages:

A. Bodily responses to extrabodily environments and 
B. cellular responses to extracellular environments (ECF, extracellular fluid). Based on what we know about ECF, (A) and (B) must not be completely separated from each other and must remain paradoxically independent of each other. In (A), ECF plays a role as a simple buffer or homogenizer of the body. Upon every external stimulus, ECF becomes more informative in cooperation with the cells responsible for muscle contraction, secretion and metabolism and lets the information on bodily response be circulated throughout the body. In (B) ECF plays a role as an environment to cells. ECF remains unconcerned about letting cells sense how the body is responding to an external stimulus. Both body and cell have an ability to build an immediate response to a stimulus. Both body and cell must be gifted with the following. (F) a force to fix a baseline during adaptation to the environment.

C. The capacity to switch off the junction to baseline on its own and deviate (be dissociated) transiently from baseline and return to (be restored to) baseline. Both body and cell need a reliable inside trigger for taking off from the baseline. We cannot be confident that we act when facing a stimulus until the body and cell can generate 'information' (trigger) of their own.

A specific molecule produced by the 'information'-generating system is required for bodily or cellular responses since a simple introduction and utilization of environmental molecule(s) as information means that information is shared between the environment and body/cell across membranes. Both body and cell must generate 'information' molecule(s) that an environment can never produce by itself. The molecule must not be a stranger to the body and cell at baseline. Body and cell cannot interpret a strange molecule as information since information must always bear messages to constantly compare a poststimulus condition to a peristimulus condition by measurements on a common scale. 'Information'-generating systems for either body or cell must function under the following rules on every occasion.

a) Independence of a baseline-fixing system. Subordination to the baseline fixed.

b) Sure, start and compulsory termination on every external stimulus.

c) Consideration for the reserve capacity of the baseline of the body and cell.

d) No requirement in the body and cell's own ATP while being produced at every start and termination.

Any cell must not have two coincidental 'information'generating systems for bodily and cellular responses since a single cell must not generate 'information' twice in response to a single external stimulus. Bodily and cellular responses must not have identical 'information'-generating systems since a never-ending circuit can be formed when an 'output' from a bodily system coincides with an 'input' to a cellular system. 'Information' that is to be generated by the body and cell must be absolutely identical to each other between the bodily and cellular systems since each cell is a ruler of the cellular system and simultaneously a subject to the bodily system. Here, we face a paradox: How can body and cell can share a single 'information' molecule across the membrane and ECF based upon an assumption that the molecule must not freely penetrate through the membrane? The paradox we face must be a clue to unraveling a true mechanism of generating 'information'.

\section{Part Three}

All the biological processes in a living system are realized in chemical reactions. Probable reaction mechanisms under biological conditions and with a biologically significant rate of reaction are roughly divided into (a) and (b) or ( and ( $\bigotimes$ )and are classified

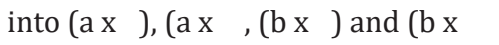

a) A protein that can recognize a specific substance at a specific site is absolutely necessary.

b) Such a protein is not necessary. ( $\mathbb{W}$ Homolytic cleavage of an electron-pair bond that can give rise to a species with an unpaired electron on the outer orbital is absolutely necessary.

( $\otimes$ Such homolytic cleavage is not necessary. (a) is required for every start of a biological process when a cell cannot expect higher acceleration or deceleration of a chemical reaction than can be attained without protein enzymes. ( $\$$ is required for every start of a biological process when a cell cannot expect higher velocity and economy than can be attained without radical species. A cell can do without consuming ATP derived from its own mitochondria by letting a reaction be initiated and propagated across the membrane. This aspect is quite important to the cells since ATP consumption at the initiation of a biological process, by itself, can be interpreted as information by the cell. $(\mathbb{Q})$ is further divided into two classes. ( $\nabla$ 1) Reaction intermediates consist of only short-lived species unable to regenerate radicals. The greatest possible acceleration is attained at the very beginning. Acceleration time is usually much shorter than deceleration time. ( $\otimes-2)$ Some of the reaction intermediates or products can regenerate radicals. The preferred reaction path of (i)-(iii) is determined primarily by an initial condition of a reaction (Figure 1).

i. Rates of reactions stay very low and remain almost constant from the moment immediately after the beginning.

ii. Rates of reactions initially increase with the greatest possible acceleration attained at the very beginning, and then the rates approach asymptotically to a constant.

iii. Rates of reactions increase robustly at the beginning and then suddenly fall to zero when either donors or acceptors of a single electron on the outer orbital are completely depleted, and acceleration time is usually much longer than deceleration time.

It seems quite difficult to distinguish ( $\nabla-2$ iii) and $(\nabla-1)$ from a kinetic point of view during the acceleration period. It is true for inflammation. An 'information'-generating system must be ascribed to a function of a unique protein. This system must be operated 
through an $(\mathrm{a} x \rrbracket)$ mechanism. The system must observe all the rules of (1)-(4) in Part two and take all the responsibilities that fall on 'information'-generating systems. For the acute beginning of inflammatory processes, at least, either ( $\bigotimes-2$ iii) or ( $\bigotimes-1)$ appears to be a probable candidate for a reaction mechanism involved in 'information' generation in the body and cell. With regard to the cells with heavy responsibilities located at the site of inflammation, an 'information'-generating system must start immediately upon the receipt of a bodily message on inflammatory stress with the shortest possible acceleration time. The system must function independently of its own ATP production from start to termination. Insufficiency of cellular ability to start and terminate an 'information'-generating system for itself can be interpreted by the cell as 'to hold it' and 'to continue it', respectively. The cell goes into a decline in the ability to respond quickly and does not respond.

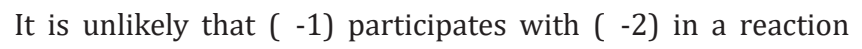
occurring at the same site, in the same cell and with the same

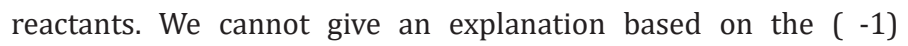
mechanism alone for the difference in a chemical basis between acutely and slowly beginning inflammatory diseases. It may well be true that a cell can choose one of ( $\bigotimes-2$ i), ( $\bigotimes-2$ ii) and ( $\bigotimes-2$ iii) according to the condition during the start and termination of the 'information'-generating system. Here, we face a question. How can a cell properly use three paths of the ( $(-2)$ mechanism to start and terminate an 'information'-generating system? What cellular factor can lead the cell to a condition where one of ( $\bigotimes-2$ i), ( $\bigotimes-2$ ii) and

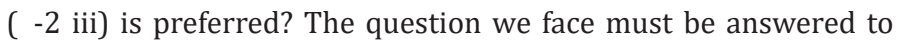
unravel the truth.

\section{Hypothesis}

\section{List of Candidate Molecules}

\section{'Information'-Generating System:}

a. Body: Tyrosine hydroxylase $\uparrow$ (increased activity)

\section{b. Cell: Monoamine oxidase $\mathrm{B} \downarrow$ (decreased activity)}

\section{Information:}

a. Body: Extracellular increasing dopamine

b. Cell: Intracellular increasing dopamine

Molecule Engaged in Terminating the System Through Negative:

a. Feedback Body: Norepinephrine

b. Cell: Endogenous I2-binding site ligands

'Messenger' across ECF:

a) L-DOPA

Indole-2,3-Dione may be a Probable Candidate for Starting and Terminating the Cellular Mao-B System

All known endogenous I2-binding site ligands are formed through decarboxylation of large amino acids. These ligands include tryptamine, serotonin and histamine [1]. The ligands are expected to decrease with an increase in L-DOPA imported to the cells during stress, leading to a rise in MAO-B activity. The degree of activation may be higher for the cells located at the site of inflammation. Indole2,3-dione is also a known endogenous I2-binding site ligand1 with MAO-B inhibitory activity [2]. However, the quinonoid is quite peculiar since it is independent of any decarboxylase activities. The quinonoid is suspected of being formed oxidatively from large amino acid phenylalanine. Evidence has been accumulating that the quinonoid may be the actual product of MAO activity. The quinonoid is increased during acute stress in parallel with an increase in L-DOPA [3-5]. The quinonoid can participate in ( $\bigotimes-2$ i), ( $\bigotimes-2$ ii) and ( $\-2$ iii) under normal conditions (without inflammation) and in the case of slowly or acutely beginning inflammatory diseases, respectively (Figure 1).

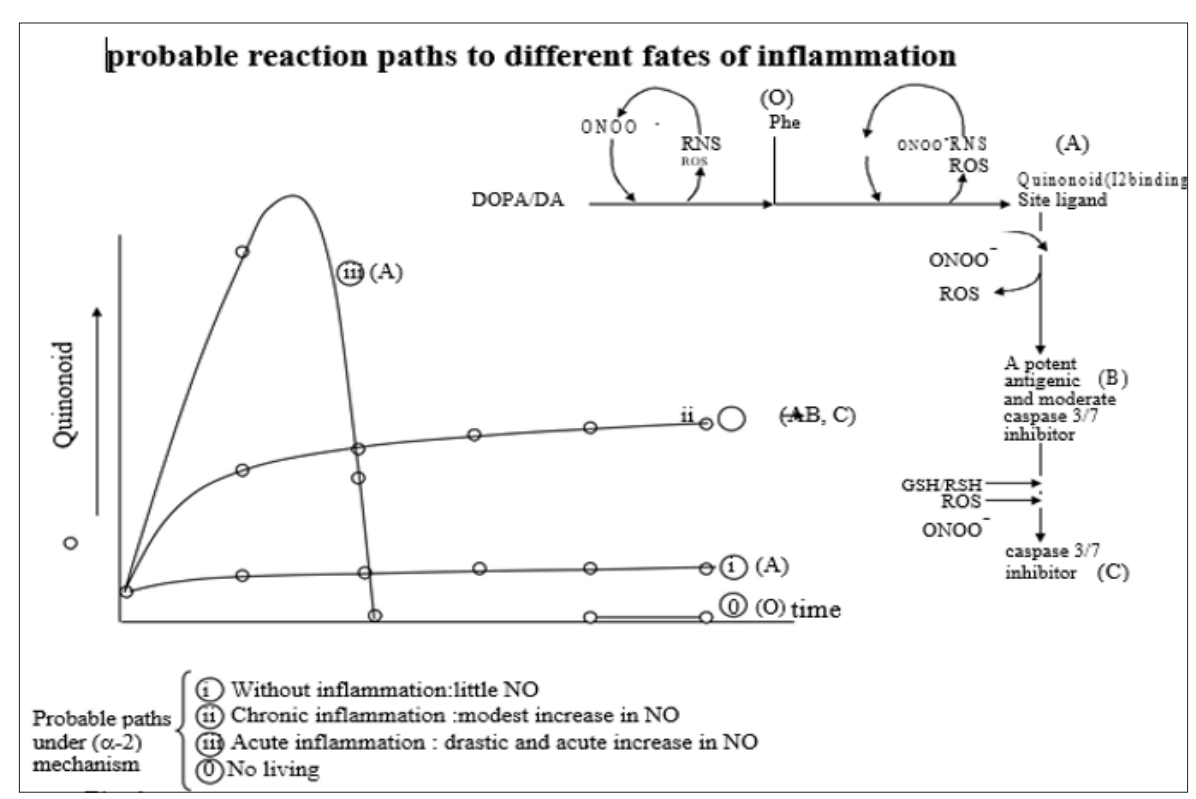

Figure 1: Probable paths to different fates of indole-2,3-dione under ( $(-2)$ mechanism. 
The quinonoid can participate in ( $\bigotimes-2$ i) under normal conditions without inflammation. This means that the basal levels of the quinonoid are fixed by the body and cell at baseline. The quinonoid can participate in $(\bigotimes-2$ ii) in slowly beginning inflammatory diseases because it is combined with peroxynitrite (ONOO-) to regenerate reactive species designated nitrogen dioxide (Figure 2). Nitro-substituted indole-2,3-dione can no longer be recognized at the I2-binding site. This outcome leads to a prolonged activation of MAO-B. Moderate increases in quinonoid production by MAO-B continues. Chronically increased quinonoid is capable of redox cycling under oxidative conditions (Figure 2). The quinonoid can participate in ( $\bigotimes-2$ iii) in acutely beginning inflammatory diseases. Acute and drastic increases in quinonoid production by MAO-B must cease when donors or acceptors of single electrons are depleted in a compartment. MAO-B activity is suppressed through the I2-binding site. The quinonoid can restore itself to its basal level.

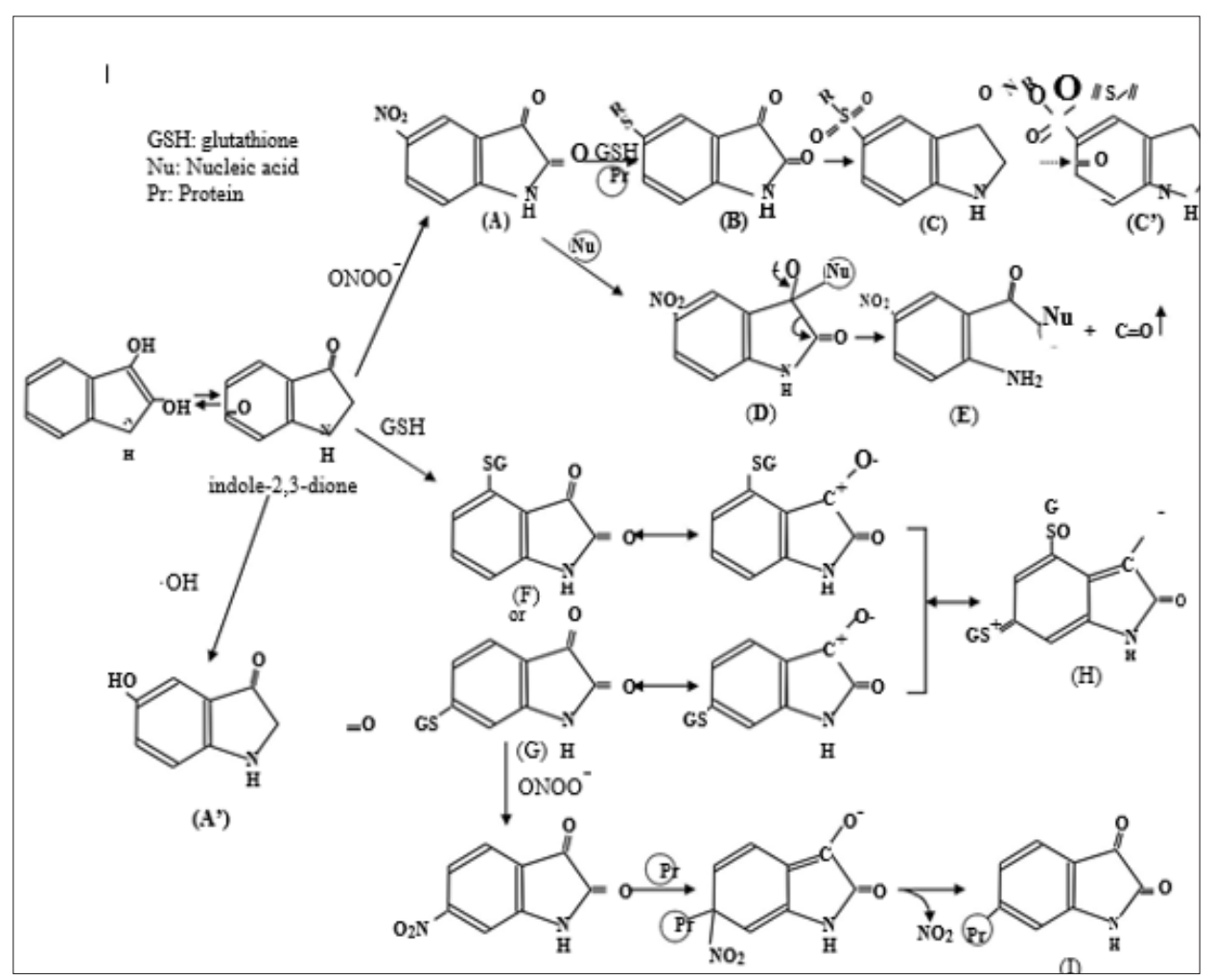

Figure 2: Schematic drawing of indole-2,3-dione and its derivatives.

\section{Testing Hypothesis}

It should be confirmed that the initial rate of increase in nitric oxide (NO) availability in a compartment is a discriminating

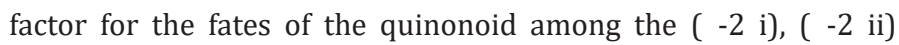
and $(\mathbb{-}-2$ iii) reaction paths. At the highest rate of increase in NO, the quinonoid is expected to participate in acutely beginning inflammatory processes through ( $\otimes-2$ iii). In the case of a modest rate, the quinonoid is expected to participate in slowly beginning processes through $(\bigotimes-2 \mathrm{ii}$ ). In the case of a slow rate, the quinonoid is expected to participate in normal inflammation-free processes through ( $\bigotimes-2$ i). It is quite important that a cell can recognize by itself how much responsibility the cell must take locally to recover from inflammation, in other words, how fast and high a cell must deviate from baseline to combat inflammatory stress.

\section{References}

1. Hudson AL, Luscombe S, Gough RE, Nutt DJ, Tyacke RJ (1999) Endogenous indoleamines demonstrate moderate affinity for I2 binding sites. Ann NY Acad Sci 881: 212-216.

2. Glover V, Halket JM, Watkins PJ, Clow A, Goodwin BL, Sandler M (1988) Isatin: identity with the purified endogenous monoamine oxidase inhibitor tribulin. J Neurochem 51: 656-659.

3. Tozawa Y,Ueki A,Manabe S, Matsushima K(1998) Stress-induced increase in urinary isatin excreton in rats: reversal by both dexamethasone and $\alpha$-methyl-p-tyrosine. Biochem Pharmacol 56(8): 1041-1046.

4. Tozawa Y, Ueki A, Shimosawa T, Fujita T (1999) 5-HT2A/2C receptor agonist-induced increase in urinary isatin excretion in rats: reversal by both diazepam and dexamethasone. Biochem Pharmacol 58(8): 13291334.

5. Tozawa Y, Matsushima K (2002) Peripheral 5-HT2a-receptor-mediated formation of an inhibitor of atrial natriuretic peptide binding involves inflammation. Eur J Pharmacol 440(1): 37-44. 
ISSN: 2574-1241

DOI: $10.26717 / B J S T R .2018 .11 .002088$

Yumiko Tozawa. Biomed J Sci \& Tech Res

(i) This work is licensed under Creative Commons Attribution 4.0 License

Submission Link: https://biomedres.us/submit-manuscript.php

$\begin{array}{ll}\text { BIOMEDICAL } & \text { Assets of Publishing with us } \\ \text { RESEARCHES } & \text { - Global archiving of articles } \\ \text { - Immediate, unrestricted online access }\end{array}$

